\title{
Rezensionen
}

Sünne Andresen, Mechthild Koreuber, Dorothe Lüdke (Hrsg.): Gender und Diversity: Albtraum oder Traumpaar? Interdisziplinärer Dialog zur „Modernisierung" von Geschlechter- und Gleichstellungspolitik. Wiesbaden: Verlag für Sozialwissenschaften 2009, $260 \mathrm{~S}$., $€ 34,90$

„Gender und Diversity: Alptraum oder Traumpaar?" lautet der Titel des Bandes zur gleichnamigen internationalen Tagung, die 2006 an der Freien Universität Berlin stattfand. Ziel der Veranstaltung war es, die Debatte um das Verhältnis von Gender (Mainstreaming) und Diversity (Management) zu intensivieren, die jeweilige Spezifik der Begriffe zu klären sowie einen Beitrag für ein besseres Verständnis der jüngsten Entwicklungen in der $\mathrm{Ge}$ schlechterforschung und Gleichstellungspolitik zu leisten. Der vorliegende Band soll die Positionen einer kontroversen Debatte bündeln und zu einer Annäherung an eine wissenschaftlich begründete „(Neu-)Orientierung im Feld der Gleichstellungspolitik“ (23) beitragen. Angesprochen sind also ForscherInnen und AkteurInnen in Politik und Wirtschaft gleichermaßen. Im Folgenden werden ausgewählte Beiträge des Bandes vorgestellt, die an den Endpunkten des Kontinuums Traumpaar/ Alptraum verortet werden können.

Die Frage, ob das Ziel einer umfassenden Gerechtigkeit durch (Gender) Mainstreaming und/oder (Diversity) Management in greifbare Nähe rückt, verneint die Historikerin Tove Soiland entschieden. Die erste der oben genannten Strategien ist für sie Ausdruck eines neoliberalen Geschlechterregimes. Der Begriff Gender, welcher nach dem Cultural Turn an einer „kulturalistischen Verkürzung“ (38) leide, habe zwar zu einer Wahrnehmung der Aufweichung vergeschlechtlicher Identitäten, jedoch nicht zu einer Minderung von Ungleichheit beigetragen. Die Geschlechterdifferenz sei nicht das Problem, vielmehr seien es die statuswirksamen Produktionsverhältnisse. Die Einfuihrung von Diversity-Ansätzen ändere nichts an dieser Wurzel des Problems von Ungleichheit. Der Soziologe Michael Meuser argumentiert ähnlich, erwähnt jedoch positive Aspekte von Gleichstellungsmaßnahmen die im Zuge von Gender Mainstreaming entstanden sind, z.B. eine größere Akzeptanz für Gleichstellungsbelange in Organisationen und einer „Professionalisierung von Geschlechterpolitik“ (95). Dies sei aber auf den Anschluss des GenderBegriffs an eine ökonomische Semantik zurückzuführen, die soziale Differenzen als Potentiale ,auf die Organisationen als Ressourcen zurückgreifen können“ (98) gefasst habe. Keineswegs verschwänden dadurch tradierte Rollenzuschreibungen, denn in diesem ökonomischen Diskurs bleiben Frauen weiterhin das Besondere und Abweichende vom männlichen Standard. $\mathrm{Ob}$ dies zur gewünschten Gleichstellung und Gerechtigkeit führt, sieht Meuser eher skeptisch. Während bei Soiland das Akteursgefuige einen statischen Eindruck ,Mächtige - Machtlose' hinterlässt, sieht Meuser die Rolle der Akteure und die Effekte ihres Tuns differenzierter. Das gemeinsame Fazit dieser beiden Beiträge zur ,Beziehungsqualität' von Gender und Diversity: Sie können als Traumpaar betrachtet werden, da sie beide aufgrund ihrer ökonomischen Logik gut harmonieren. Für die gesellschaftliche Bearbeitung von Ungerechtigkeit sind sie jedoch eher ein Alptraum. 
Ebenfalls kritisch diskutiert die Politikwissenschaftlerin Claudia von Braunmühl in einer „Gewinn-und-Verlust-Rechnung“ das Verhältnis zwischen Gender Mainstreaming und Diversity Management. Im Gegensatz zu Soiland hebt sie Gender Mainstreaming (GM) als eine Institution hervor die auf der Forderung der internationalen Frauenbewegung nach Gerechtigkeit basiere. „Ein nicht hinzunehmender Verlust“ wäre es aber, wenn Diversity Management (DiM) ,den immer noch aktuellen Anspruch von Geschlechtergleichheit und -gerechtigkeit“, den GM begründe, ,entwerten würde." (63) Die Konsequenz wäre also ein ernster Streit zwischen den ,Partnern' und dieser scheint nicht unwahrscheinlich, da beide in ihren Grundfesten zu verschieden sind.

Die Ethnologin Susanne Schröter würdigt hingegen Diversity als „,radikales Bekenntnis zur Globalisierung und Moderne“ (79), auch wenn es sich dabei mitunter um eine Unternehmensstrategie handele. Für sie schwinge bei Diversity die Forderung nach „Toleranz, Anti-Diskriminierung und Realisierung von Chancengleichheit" mit. Am Beispiel der Kategorie Gender, die für Schröter die Begriffe „Frau“ und „Mann“ integriere und gleichzeitig destabilisiere (80), möchte die Autorin aufzeigen, wie der „Kampf um Teilhabe“ in Theorie und Praxis ,zur Verabschiedung eines homogenisierenden Modells und zur Anerkennung von Diversität" führe (79). Sie versucht damit einen Einblick in die hochkomplexe Geschichte des Gender-Begriffs zu geben und gleichzeitig Anknüpfungspunkte mit Diversity aufzuspüren. Pragmatisch für eine „Vernunftehe“ eintretend betrachtet Gertraude Krell, Professorin fur Betriebswirtschaftslehre, die Verbindung der konkurrierenden „Konstrukte oder Konzepte“ Gender und Diversity, da ,immer schon eine Verbindung" zwischen diesen bestanden hätte. Weiterhin sei es angemessen, der polarisierten Diskussion die ,emotionale Heftigkeit" (133) zu nehmen. Sie untermauert ihr Argument mit drei empirischen Befunden, welche verdeutlichen sollen, dass ,mit Blick auf Diskriminierung und Ausgrenzung" (136) Gender nicht das einzig wichtige Merkmal sei. Ein Königsweg in der gleichstellungspolitischen Umsetzung, also ob Diversity unter dem Dach Gender oder Gender unter Diversity zu etikettieren ist oder ob vielmehr keine Hierarchisierung zwischen Gender und Diversity vorgenommen werden soll, gäbe es nicht. Dies zeige sich auch in der Praxis. Hier könne nicht von typischen linearen Entwicklungsmustern im Sinne eines ,weg von“ z.B. klassischer Frauenförderung ,hin zu“ Diversity Management gesprochen werden. Die Politikwissenschaftlerinnen Barbara Riedmüller und Dagmar Vinz diskutieren die Relevanz von Gender und Diversity in der Sozialpolitik- und Wohlfahrtsstaatforschung und fordern, die historischen Wurzeln und Besonderheiten beider Kategorien anzuerkennen. Ihr Beitrag fokussiert die Sozialund Arbeitsmarktpolitik, ein Feld, dass Soiland mitunter implizit als zentrales strukturelles Problem betrachtet. Insbesondere der Entstehungskontext von Diversity in der Bürgerrechtsbewegung der USA, den Günther Vedder ausfuihrlich in seinem Beitrag behandelt, werde häufig verkannt. Schnittmengen und Potentiale von Gender und Diversity in der Forschung zu erkennen, kann zum „Nachdenken über Zukunftsfragen der Gesellschaft" und zum „Entwickeln von Perspektiven für eine neue Architektur der sozialen Sicherung" anregen (65).

Schon in der Auswahl der AutorInnen wird die Stärke des Sammelbandes mit dem provokanten Titel deutlich: seine Interdisziplinarität und die Sicht auf das Spannungsfeld ,modernisierter" Gleichstellungspolitik und der ihr zugrunde liegenden Konzepte Gender und Diversity. Der Leserin bietet sich ein Spektrum 
mannigfaltiger, wissenschaftlich kontroverser Blickwinkel. Die Spannbreite der Diskussion ist eine Stärke des Sammelbandes. Diese kann jedoch auch als eine Schwäche bezeichnet werden. Die thematische und konzeptionelle Breite innerhalb einiger Texte erschwert es LeserInnen ohne Vorwissen in der Frauen- und Geschlechterforschung, die jeweils zentralen Punkte herauszuarbeiten sowie eine sinnvolle Verknüpfung der Konzepte Gender und Diversity zu erkennen. Zudem fällt auf, dass einige Beiträge - Fallstudien zum Transfer feministischer Theorie in die Praxis - Fragen zu Diversity nicht bzw. nur indirekt (Meyerson \& Kolb; Meriläinen et al.) behandeln.

Ein Fazit des Bandes könnte lauten: Eine pauschale Antwort zur ,Paarbildung erscheint unmöglich. Die Situationen, in denen entweder ein erstes vorsichtiges ,Dating', eine Ehe oder eine Trennung von Gender (Mainstreaming) und Diversity (Management) angemessen sein könnte, sind an komplexe strukturelle Zusammenhänge gebunden in denen vielfältige Akteurinnen und Akteure mit unterschiedlichen Interessen mitwirken.

\section{Eva Wegrzyn}

Rainer Bartel/Ilona Horwarth/Waltraud Kannonier-Finster/Maria Mesner/Erik Pfefferkorn/Meinrad Ziegler (Hrsg.): Heteronormativität und Homosexualitäten. Innsbruck: Studienverlag, 2008, 152 Seiten, $€ 14,90$

Der Band „Heteronormativität und Homosexualitäten" geht auf eine gleichnamige Tagung zurück, die 2006 in Linz stattfand und damals mit dem Zusatz ,Forschung in Anknüpfung an Michael Pollak' untertitelt war. Auf diese Kontextualisierung wird in dem Sammelband lediglich in der ,Entstehungsgeschichte' von Rainer Bartel explizit Bezug genommen: Michael Pollak, 1948 in Wien geboren, verließ Österreich nach seinem Soziologie-Studium in Linz, um in Paris - zunächst bei Pierre Bourdieu - seine Forschungen zu realisieren. Mit der Tagung sollte der Versuch unternommen werden, den 1992 in Paris verstorbenen Forscher bzw. den Geist seiner Forschung nach Linz zurückzuholen: Die Tagung ,sollte... seinen Blickwinkel einnehmen und seine Arbeitsweise aufnehmen" (10).

Wozu genau Michael Pollak forschte, wird in dem Sammelband, der sich als Auswahl und Weiterentwicklung der Tagungsbeiträge versteht, nicht weiter ausgeführt. Erwähnt wird aus dem breiten Spektrum an Forschungsinteressen die „empirische Erforschung homosexueller Milieus im Spiegel der herrschenden gesellschaftlichen Verhältnisse" (9), an die hier angeknüpft wird. In diesem Sinne kann die kritische Analyse heteronormativer Strukturen und homosexueller Politiken als Verbindungslinie zwischen den Beiträgen angesehen werden, die ansonsten hinsichtlich ihrer theoretischen Bezüge und gesellschaftlich-politischen Verortung eher disparat erscheinen.

Die Idee, Michael Pollak in Form einer Tagung zu gedenken, wie auch die Fortführung der Diskussionen im Rahmen eines Sammelbands wurde als Kooperationsprojekt zwischen der Homosexuellen Initiative HOSI Linz, dem Institut für Frauen- und Geschlechterforschung und dem Institut für Soziologie der JKU Linz realisiert. Dieser Verbund liegt an der Schnittstelle von wissenschaftlicher Produktion und sozialer Bewegung: Anliegen der HOSI war, ,ein Zeichen der Sichtbarkeit für homosexuelle Lebensformen in einem anerkannten Feld der kulturellen und politischen Öffentlichkeit“ (7) zu setzen, so die HerausgeberInnen im Vorwort. Von Interesse ist nun, wie sich dieses ,Zeichen 
der Sichtbarkeit' in dem Sammelband artikuliert und - im Anschluss an den Titel - wie „Heteronormativität und Homosexualitäten " aufeinander bezogen diskutiert werden.

In seiner ,Einleitung: Heteronormativität und die Verflüssigung des Selbstverständlichen" resümiert Meinrad Ziegler zunächst die theoretischen Kontexte, die dazu beitragen, ,dem Prinzip der Zweigeschlechtlichkeit die Selbstverständlichkeit zu rauben" (17). Er verweist auf zentrale Arbeiten, Analysen und Erkenntnisse in der Frauen- und Geschlechterforschung sowie der Queer Studies, scheint dabei allerdings die „dauerhafte Identifizierung mit einem Geschlecht" (20) einem emphatisch besetzten Begriff von Veränderung, Verflüssigung und Vielfalt gegenüber zu stellen. Diese Dimensionierung - des Normativen, Feststehenden auf der einen und der Vervielfältigung auf der anderen Seite - wird in den folgenden Beiträgen thematisiert und kritisch bearbeitet.

So erinnert Alice Pechriggl in ihrer Analyse „Naturrechtliche ,Heteronormativität' vs. politische Normsetzung" daran, dass es ,ursprünglich gerade die Setzung war, welche die Norm als Gesetz (von nomos) im Gegensatz zur Gewachsenheit bzw. der Unabänderlichkeit der Natur (physis) auszeichnete" (25). Vor diesem Hintergrund kritisiert sie eine Tendenz im Anschluss an Butlers Heteronormativitätskritik, die „... Normativität gleichsam naturalisiert, indem sie zu einer transzendentalen, aber zugleich empirischen Verfasstheit gemacht wird" (26). Auch wenn diese Kritik an der Rezeption Butlers durchaus streitbar ist, so findet der Anspruch, den Begriff der Normativität aus der Perspektive ,einer reinen Rechtslehre" (26) zu betrachten - und damit den Aspekt der (sozialen) Setzung in den Vordergrund zu rücken -, eine komplexe und anregende Umsetzung.

Einen Kontrapunkt zu Pechriggls Analyse positiver Normativität - im Sinne „von der Mehrheit anerkannt" (40) - setzt Antke Engel in ihrem Beitrag "Gefeierte Vielfalt. Umstrittene Heterogenität. Befriedete Provokation.“ Nach der ,Toleranz kapitalistischer Verhältnisse für nichtnormgerechte sexuelle Identitäten“ (48) fragend, entwickelt sie den Begriff der projektiven Integration, die die Norm selbst pluralisiere: „Bilder hybrider, flexibler und ambivalenter Identitäten werden als Inbegriff erfolgreicher, kreativer Individuen projektiv aufgeladen" (52). Als Konsequenz aus dieser Analyse zielt ihr Plädoyer einer "Politics of. Strangeness“ (55) darauf, „die Prozesse normativer Differenzproduktion zu unterbrechen, über die sich Dominanzverhältnisse reproduzieren" (56f.). In ihrer differenzierten Analyse über eine klare Hetero-/HomoOpposition hinausgehend, fragt sie nach Konzepten und Praxen des Sexuellen, die aufgezeigte neoliberale Transformationen kontern könnten.

Wenn aus diesem Blickwinkel queerer Ökonomiekritik Hetero- und Homonormativität durchaus eine Allianz eingehen können, so konzentriert sich Phil C. Langer in "Paradoxes Begehren“" wiederum eindeutig auf heteronormative Männlichkeitsbilder und ihre Bedeutung in der Psychodynamik von HIVNeuinfektionen. Er kontert den immer noch aktuellen „Topos von Aids als Preis, den der Schwule für die Übertretung gesellschaftlicher Normen zu zahlen habe“ (69) mit einer Umkehrung: „Heteronormative Männlichkeitsbilder stellen einen ernst zu nehmenden Risikofaktor innerhalb schwuler Lebenskontexte dar" (77). Auch wenn fraglich bleibt, ob die Suche nach Kausalzusammenhängen und lineare Erklärungsmodelle weiter führen, so ist der Verweis auf eine ,im öffentlichen Diskurs behauptete gesellschaftliche Akzeptanz von Homosexualität" (79) durchaus bedenkenswert. 
Dieser Linie folgt Christoph Treiblmayr in diskursanalytisch angelegten Überlegungen zu männlichen Homosexualitäten im deutschen Kino der 1990er Jahre. In seinem Beitrag „,Von ,bewegten Männern' und ,queeren' Gender-Utopien“" greift er aus den Rubriken ,Neue Deutsche Beziehungskomödie' und ,New Queer Cinema' je einen Film heraus: „Der bewegte Mann" macht deutlich, dass das gleichgeschlechtliche Begehren keinen AusschlieBungsgrund mehr darstellt, solange „,die Figur des besten schwulen Freundes" (91) sich der zugedachten Rolle entsprechend verhält und in der heterosexuellen Liebesbeziehung „als Katalysator“ (99) fungiert. Ganz anders „Prinz in Hölleland“, der sich ,beharrlich weigert, positive Bilder von Homosexualität zu zeigen oder Identifikationsangebote für die RezipientInnen zu machen" (102) - sich in diesem Sinne also einer Beteiligung an der Produktion normativer Bilder entzieht.

„Für eine neue Grammatik der Anerkennung" plädiert denn auch Christine $M$. Klapeer, wenn sie danach fragt, ,wie und nicht nur dass von einer Anerkennung lesbischer und schwuler Existenzweisen gesprochen wird" (110). Dem intersubjektiven Ansatz Honneths stellt sie das Modell „Partizipative Parität“ von Fraser gegenüber: „haben wir es innerhalb der Lesben- und Schwulenbewegungen doch mit einigen ... problematischen Anerkennungsdiskursen $\mathrm{zu}$ tun, welche stark auf Selbstverwirklichung, gleiche Rechte und individuelle Anerkennung fokussiert sind“ (115). Sie rekurriert auf „feministische Theorien und Bewegungspraxen als kritisches Korrektiv“ (117) und plädiert mit Fraser für ,eine doppelte Perspektive“ (121): auf staatliche Politiken, Institutionen und demokratische Prozesse wie auch auf kritische Arbeit innerhalb LGBT-Politiken selbst.

Diese Selbst/Kritik vollzieht Sushila Mesquita, wenn sie in „Heteronormati- vität und Sichtbarkeit" die Ambivalenzen von Sichtbarkeit aufzeigt und danach fragt, ,unter welchen Voraussetzungen, mit welchen Konsequenzen und auf welche Arten welche Lebensentwürfe derzeit öffentliche Sichtbarkeit erlangen können" (129). Anhand von zwei US-amerikanischen Sitcoms, die im ORF ausgestrahlt wurden, veranschaulicht sie zwei Formen des Othering: Normalisierung als „Anpassung mit Abstrichen" und Karnevalisierung als „Zuschreibung des Status eines konstitutiven Außen“ (137). Diese „Kopplung von Sichtbarkeit an normative Konventionen der Lesbarkeit" (144) betonend ruft die Autor_in dazu auf, das komplexe Verhältnis von Sichtbarkeit und politischer Macht differenziert wahrzunehmen und statt eines ,Mehr' an Sichtbarkeit gängige Darstellungsformen und Lesarten herauszufordern.

Die Schnittstelle von Wissenschaft und sozialer Bewegung ist dem Band deutlich anzumerken: Das Ringen um Sichtbarkeit nicht-heterosexueller Lebensweisen geht einher mit der notwendigen Reflektion ihrer Einbindung in dominante Bewertungsmuster. In ihrer Disparität setzen sich alle Beiträge mit dem Konzept des Normativen auseinander, wobei der Bogen hin zu einer kritischen Analyse des Ineinandergreifens neoliberaler Entwicklungen und sexueller Politiken verläuft. Der selbst/kritische Bezug auf Identitätspolitiken verhindert dabei eine Übersetzung von homosexuell in schwul, so dass Differenzierungen entlang verschiedener Machtachsen Raum bekommen. Es ist eine kritische Momentaufnahme, die in eindrücklicher Weise das Spannungsfeld aufzeigt, in dem heteronormativitätskritische Arbeiten sich bewegen.

Karen Wagels 
Marina Gržinić/Rosa Reitsamer (Hrsg.): New Feminism. Worlds of Feminism, Queer and Networking Conditions. Wien: Löcker Verlag 2008, 470 S., € 29,80 Melanie Groß: Geschlecht und Widerstand. post..। queer.. I linksradikal. Königstein/Taunus: Ulrike Helmer Verlag 2008, 249 S., € 19,90

Was Feminismus, was feministische Politik bedeutet, die historischen Strategien, Debatten, aufgehobenen Hoffnungen und Diskurs-umschifften „Vorsicht"-Schilder: vieles von alledem kann eine kennen. Trotzdem oder deswegen: Wie bringen Feministinnen "heute“ das Wissen, das Denken, das Handeln zusammen? Welche Feministinnen? Wo? Die beiden zu besprechenden Bücher kamen etwa zeitgleich mit den deutschen Bestsellern des angeblichen Neofeminismus à la „Alpha-Mädchen “ auf den Markt. Ihre Ambition, eine feministische Gegenwart so zu reflektieren, dass dies politisch (und) optimistisch in die Zukunft weist, verbindet die zwei Bücher. In anderer Hinsicht könnten sie unterschiedlicher kaum sein. Bei Melanie Groß' Monografie „Geschlecht und Widerstand“ handelt es sich um die Drucklegung einer Dissertation mit durchgängig sozialwissenschaftlichem Referenzrahmen hinsichtlich Analyse und Methodik, um einen dementsprechend geschlossenen Aufbau der Argumentation und um eine ganz lokale Fokussierung. Der von Marina Gržinić und Rosa Reitsamer herausgegebene Sammelband "New Feminism" versammelt einen Mix an Genres, bei dem Interviews und journalistische Texte überwiegen, um durchmischte Zugänge und Disziplinen und insgesamt um einen höchst globalen Anspruch der Herausgeberinnen.

„New Feminism. Worlds of Feminism, Queer and Networking Conditions" ist ein schwer gewichtiges, umfangreiches, eher nur portionsweise er-lesbares Werk mit über 40 Beiträgen, die in zehn Ab- schnitten organisiert sind, von „We Are (Be)coming!“" über „Forms of Anti-/Precarization" bis "Strategic Agencies“. In der Einleitung der Herausgeberinnen präsentiert sich der Band nicht allein als eine Sammlung von Texten zur Reflexion gegenwärtiger feministischer Positionen und Praxen, sondern weit anspruchsvoller als Plattform zur Vorstellung von ,new actors, agents and forces who do not talk only about unknown histories, but first and foremost re-articulate the very foundation of what the feminist movement is" (13). „New Feminism“, erklären die herausgebenden Künstlerinnen und Kulturwissenschaftlerinnen aus Wien und Ljubljana, ,is a term that tries, firstly, to break the simple continuity in the feminist movement and, secondly, to re-engage new agencies and topics within the movement"(13). Ihre Beiträger_innen (fast ausschließlich: ,-innen") waren aufgefordert ,to propose a politically engaged break with the one and only history and present of feminism"(14).

Ein vollmundiger Anspruch also, der auf seine Einlösung umso neugieriger macht, als doch das, was hier als ,neu“ bezeichnet wird - in erster Linie eine Dezentrierung der Metaerzählung des Feminismus -, integrierender Bestandteil der so genannten Third Wave war oder ist und jedenfalls zu guten Teilen in den späten 1980er Jahren formuliert wurde. Wer also war eingeladen, die eigene Stimme in eine Polyphonie des Diskontinuierlichen zu tragen, und welche minoritären Geschichten kommen zu Wort?

Die Abschnitte, mit knappem SammelAbstract versehen, öffnen jeweils mit einem Interview mit Trägerinnen eines „Big Name“, darunter Braidotti, Butler, Spivak, Trinh. Das Gespräch mit Judith Butler fokussiert auf ihr Buch „Precarious Life“" und gegenwärtige queere Bewegungen, Gayatri Spivak rekapituliert eine Vielzahl ihrer Publikationen und Begriffsprägungen, von Subalternität bis „cultural trans- 
lation", und Evelyn Tornton Beck schließlich äußert sich sehr positiv über die zunehmende Akzeptanz von Homosexuellen in jüdischen Gemeinden in den rund $25 \mathrm{Jah}$ ren seit ihrem Buch „Nice Jewish Girls“. Trinh T. Minh-Hd sagt, ganz wie schon in ihren Schriften von vor zwanzig Jahren, dass es genuin neue Denk- oder Schreibweisen gar nicht geben könne, sondern stets nur Verlagerungen, neue Kombinationen, neue Blicke auf die gleichen Bilder oder Objekte. „Post“--, ,Trans“- und „Avant"- oder „Retro"-Metaphern suggerierten eine chronologische Ordnung, die adäquater doch als spiralförmig vorzustellen sei.

Die Zusammensetzung der Autor_innen sollte wohl kulturelle, postkoloniale, politische Vielfalt realisieren, orientierte sich zu Teilen aber offenbar eher am Kriterium der Erreichbarkeit als an einer umfassenden Auswahl impulsgebener Akteur_innen und Denker_innen ,weltweit". Namen verdienstvoller deutscher Autorinnen befinden sich darunter, wie Birgit Rommelspacher, Antke Engel, Yvonne Doderer. Österreichische Autor_innen sind zahlreicher präsent, als inhaltlich vielleicht zu überzeugen vermag. Künstler_innen und Kunsttheoretiker_innen figurieren prominent, etwa Tanja Ostojićs mit einem Bericht über ihre verschiedenen „Border Crossing"-Projekte oder das rumänische Kollektiv H.arta. ,Osteuropa“ ist mit Beiträger_innen insbesondere aus Ländern des ehemaligen Jugoslawien stark vertreten, was sich auch daraus erklären dürfte, dass an einem Anfang des Projekts eigentlich ein „West/Ost-Europa“-Buch anvisiert war (vgl. 12). Natasa Velikonja schreibt über „Homo-Konservatismus" und die Depolitisierung der Lesben- und Schwulenbewegung in Slowenien, Đurđa Knežević über Institutionalisierungs- und Rückzugstendenzen in der Frauenbewegung in Kroatien, Rutvica Andrijasevic über Bilderpolitik in Anti-Trafficking-Kampagnen der Inter- national Organisation for Migration. Nataša Govedić präsentiert sehr spannende künstlerische Recherchen zu Körperwahrnehmungen und Körper-,,Zeugnissen“. Biljana Kašić schließlich zieht aus der Vielfältigkeit von Feminismen ähnliche Schlüsse wie Trinh:

„On the one hand, feminism(s) can be considered as a constant process of formation and invitation in terms of women's issues and agencies by constantly dialoguing with the theoretical-activist aspirations of different ,schools of feminist thinking' (...) and different historical points of departure; on the other hand, it seems to be a dispersive map of topographies and processes that have gone both simultaneously and in historical shifts, time-lags and discrepancies being exposed to immense shifts across global-local contexts." (455f.)

Wie kommt das Neue in die Welt (der Feminismen)? Wann begann es? Worin genau besteht es? „New Feminism“ formuliert die Verheißung, uns dazu eine ganz neue Topografie zu zeigen. Ob der Band sie einlöst, stelle ich dem Urteil der Leserin anheim.

Die in Bielefeld approbierte Promotion der Soziologin Melanie Gro $\beta$, „Geschlecht und Widerstand. post..| quer..| linksradikal..", formuliert kein so weltumspannendes Versprechen. Sie erklärt drei feministisch-queere Gruppen einer ,mittelgroBen Stadt" (Deutschlands, wie wohl zu ergänzen wäre) für zentral - eine Setzung, deren Dezentrierung ihr, um diese Kritik vorwegzunehmen, in den theoretischen Ausfuhhrungen teilweise, im Empirischen hingegen weniger gelingt.

Gro $\beta$ interessiert sich für die Bewegung von Akteur_innen auf jenem widersprüchlichen Feld, das von der (dekonstruktivistisch-queer motivierten) Ablehnung geschlechtlicher Zuschreibungen abgesteckt ist und von der Achtung vor jener Gewalt, die sich mit Ein- und Ausschlüssen verbindet; ein Feld, das dabei vom Begeh- 
ren nach politischem Engagement, nach Attackieren von Herrschaftsverhältnissen durchzogen bleibt. Auszugehen sei von einem Streit um Hegemonie innerhalb des Feminismus, nämlich darum, ob sozioökonomische Ungleichheitsverhältnisse oder das Symbolische und Kulturelle Vorrang hätten. Die Studie nimmt die Perspektive ein, dass dieser Hegemoniekonflikt ,tatsächlich nicht völlig lösbar ist, gleichwohl aber nicht zwingend ein Entweder-Oder produzieren muss" (12).

In einem kompetenten und konzisen Theorieteil stellt Groß aktuelle feministische Positionen dar - von der Kritischen Theorie bis zur Critical Whiteness - und erläutert dann feministische Epistemologien sowie Gründzüge der Grounded Theory, mit der sie selbst arbeitet. Die Erforschung der Ränder des feministischen Diskurses (anstatt seines Mainstreams), so betont sie, ermögliche nicht nur spezifische Einsichten, sondern auch generell eine Hintanhaltung von Schließungsprozessen. Entsprechend dem "theoretischen Sampling“" der Grounded Theory wertete sie Gruppendiskussionen mit drei Formationen aus der Autonomen Szene der Stadt ihrer Wahl aus. Alle drei befassen sich mit Fragen von Geschlecht, Sexualität, Queerness und Transgender; über ihre politischen Strategien sind sie sich uneins.

Als „postfeministisch“ könne eine dieser drei gelten: hochschulpolitisch aktive Frauen, die sich in erster Linie um das Problemfeld „Normativität“ grupppieren und immer wieder an den Möglichkeiten der eigenen Agency zweifeln. Ihre Widerstandsaktionen bestehen vielfach in symbolischen Interventionen; „Spaß“ muss dabei sein. Sie beteiligen sich an Grenzcamps und unsichtbarem Theater und irritieren bei Kundgebungen in ,pink \& silver". Bei der zweiten, als "queer-feministisch" titulierbaren Formation handelt es sich um eine Musikband, die in ihrem Gruppennexus das Problem der identi- tären Zuschreibungen und Anrufungen, den Protest gegen geschlechtliche Kategorisierung also, zentral setzt. Lebenswelt und Widerstand erscheinen hier als eng verzahnt, alternative Lebensentwürfe gelten als machbar, und zu den Mitteln des Widerstandes zählen für diese Gruppe Aktionen wie Drag-Auftritte und, naheliegenderweise, das Texten und Performen von Musik. Radical Cheerleading etwa gehört ebenfalls zu den politischen Ausdrucksformen ihrer Wahl. Melanie Groß' dritte Frauengruppe versteht sich als ,linksradikal-feministisch“. Diese Aktivistinnen verwenden am ehesten die $\mathrm{Ka}$ tegorie Frau als Selbstbezeichnung, wobei der Gruppennexus auf dem Problemfeld "Wirkmächtigkeit" liege: Wirkmacht des Geschlechterdualismus, Wirkmacht politischer Ausdrucks- und Widerstandsformen, wirksame Kapitalismuskritik. Zu ihren Protestformen gehören klassische Medien der Öffentlichkeitsarbeit wie Flugblätter und Demos, sie setzen auf den Ausbau subkultureller Zusammenhänge im Autonomen Zentrum und treten zudem für den Erhalt von Frauenräumen ein.

Die Verfasserin der Studie resümiert diese unterschiedlichen Diskurs- und Widerstandsformationen, wie sie vor dem Hintergrund eines je differenten Verständnisses von Macht auch verschiedene Herrschaftskonfigurationen attackieren, positiv:

"So gelingt es der untersuchten Szene, die Ebenen der symbolischen und der strukturellen Ungleichheit nicht duch ein gemeinsames in sich geschlossenes Konzept zu verbinden, sondern vielmehr durch den Einsatz dreier Interventionsarten, die auf verschiedene Angriffsziele gerichtet sind: Die Interventionen auf der Ebene der Repräsentation, die Intervention durch Skandalisierung \& Sichtbarmachung und die Interventionen durch Information \& Aufklärung." (226)

Alles paletti (wie eine frühere Generation sagen würde)? Die wohl nicht nur 
sprachlichen Hilflosigkeiten der jungen, der "neuen" Feministinnen werden in den wörtlichen Interviewpassagen, im „so, naja, irgendwie“ immer wieder deutlich. Die Verfasserin nimmt diese nur halb fertig scheinenden Bemerkungen berührend ernst, über-interpretiert sie manchmal wohl auch. Ich hätte gern noch mehr zu den politischen Praxen gelesen, dafuir vielleicht weniger Ausfuhrliches zu den methodischen Auswertungsschritten. Mir fehlten Überlegungen zur Positionalität der untersuchten Akteur_innen, beispielsweise hinsichtlich Alter und Schicht. Sie scheinen alle ,jung “ und vergleichsweise bildungsprivilegiert, und es bleibt ganz offen, ob ihre Wahrnehmung von Politik und Geschlecht damit zusammenhängen könnte.

Die Lektüre dieser beiden Bände zeitigt jedenfalls als Effekt die Überzeugung, dass Feminismen höchst lebendig bleiben. Sie mögen nicht immer an ihre Geschichte denken, ihre Reflexion entspricht nicht allen Maßstäben der Perfektion, ihre Taktiken aber scheinen tatsächlich immer wieder neu und (doch) politisch, augenfällig wie in den Demoblocks der tute bianche, pink \& silver, Women in Black ... Grau, sagt Trinh T. Minh-Hà in "New Feminism“, sei als Farbe der unendlichen Möglichkeiten zu begreifen.

Hanna Hacker
Karin Jurczyk/Mechthild Oechsle (Hrsg.): Das Private neu denken. Erosionen, Ambivalenzen, Leistungen. Münster: Verlag Westfälisches Dampfboot 2008, 332 S., $€ 29,90$

Paula-Irene Villa/Barbara Thiessen (Hrsg.): Mütter - Väter. Diskurse, Medien, Praxen. Münster: Verlag Westfälisches Dampfboot 2009, 341 S., $€ 34,90$

Das Private wird in den letzten Jahren verstärkt öffentlich und medial verhandelt. Darüber hinaus ist es zunehmend politischen Eingriffen und Regulierungen ausgesetzt. So lautet der Ausgangsbefund des von Karin Jurczyk und Mechtild Oechsle herausgegebenen Sammelbands (J./Oe.), der das Private in seinen verschiedenen Facetten und in seinem Verhältnis zum Öffentlichen neu denken will. Dieses Verhältnis ist in Bewegung geraten und mit Veränderungen verbunden, die für die Herausgeberinnen in dem Begriff der Entgrenzung zusammenlaufen: Grenzverschiebungen aufgrund Strukturveränderungen in der Erwerbsarbeit sowie veränderter privater Lebensführungen und Geschlechterbeziehungen, neuer Informations- und Kommunikationstechnologien und einer voranschreitenden Mediatisierung des Privaten bei gleichzeitiger Domestizierung des Öffentlichen, schließlich auch Grenzverschiebungen in der Frage nach rechtlichen und politischen Eingriffen in das Private. Ein Themenfeld in diesem Zusammenhang, das nur vermeintlich allein dem Privaten zuzurechnen ist, ist Elternschaft. Auch hier muss konstatiert werden, dass Bewegung in die medialen Bilder und alltäglichen Praxen von Mutterschaft und Vaterschaft gekommen ist. Der von PaulaIrene Villa und Barbara Thiessen herausgegebene Sammelband (V./T.) zum Thema Mütter und Väter hat zum Ziel, die nicht immer ganz so neue - aber gleichwohl unübersehbare - Vielfalt von Elternschaften auszuleuchten, und zwar sowohl mit Blick 
auf Praxen von Mutter- und Vaterschaft in ihrer strukturellen Eingebundenheit als auch auf mediale Diskursivierungsweisen und Verarbeitungsformen. Insofern lässt sich der Band von Jurczyk/Oechsle mit seiner grundlegenden Perspektive gewissermaßen als Rahmung für das enger gefasste Thema Elternschaften von Villa/Thiessen lesen.

Ein zentraler Fokus liegt dabei auf der Frage nach dem aktuellen Verhältnis von Öffentlichkeit und Privatheit bzw. von Erwerbsarbeit und Familie. Diese beiden Verhältnisbestimmungen gehen nicht unmittelbar ineinander auf, gleichwohl sind beide zentral mit Fragen nach geschlechtsspezifischer Arbeitsteilung und Ungleichheit, nach Differenz und Hierarchie verbunden. Die historisch geronnene Trennung der Sphären Öffentlichkeit/Erwerbsarbeit und Privatheit/Familie samt ihrer geschlechtsspezifischen Zuordnung ist für die Frauen- und Geschlechterforschung eine der zentralen Referenzfolien. Bea Lundt (in J./Oe.) rekonstruiert diese Dichotomie in ihrem Beitrag als ein Geschichtsbild mit eigener Wirkmächtigkeit, macht aber ebenso auf die Differenziertheit historischer Verhältnisse aufmerksam, die sich diesem klaren Schema zuweilen entziehen. Gleichwohl ist das generalisierende Bild getrennter Geschlechterwelten als historischer Bezugspunkt und als Deutungsmuster notwendig, um Verschiebungen im Verhältnis des Privaten zum Öffentlichen analytisch in den Blick zu bekommen. Solche Verschiebungen sind Thema einer ganzen Reihe von Beiträgen. Deutlich wird darin die Gleichzeitigkeit unterschiedlicher Entwicklungsdynamiken wie auch die Heterogenität der damit verbundenen Einschätzungen.

Eine Entwicklungsrichtung lässt sich als das Ausgreifen des Privaten ins Öffentliche beschreiben. Krishan Kumar und Ekaterina Makarova (in J./Oe.) zeigen dies in ihrem Beitrag unter anderem anhand veränderter
Verhaltensgewohnheiten im öffentlichen Raum aufgrund neuer Informations- und Kommunikationstechnologien. Das Mobiltelefon beispielsweise wird zum Vehikel, mit dem Privates als Privates in der Öffentlichkeit kommuniziert wird. Dagegen fragt Beate Rössler (in J./Oe.) nach den Gefährdungen der informationellen Privatheit durch eben diese Technologien - durch Erfassung und Speicherung persönlicher Daten beim Surfen im Internet oder im Rahmen staatlicher Überwachung - und diskutiert die Gründe, warum es dagegen nur so geringen gesellschaftlichen Widerstand gibt. Ralph Weiß (in J./Oe.) wiederum befasst sich in seinem Beitrag mit der Mediatisierung des Privaten, genauer mit der freiwilligen Veröffentlichung und Inszenierung von Privatheit im Fernsehen, welche einer „Kultur der Schamlosigkeit“ (ebd., 186) Vorschub leiste und damit persönliche Autonomie als Basis von Privatheit angreife. Demgegenüber argumentiert Helga Krïger (in J./Oe.) in ihrem Beitrag, dass das Private - hier nun allerdings verstanden als Oberbegriff für familiale Zusammenhänge - zunehmend in das Öffentliche hineinragt und den dort abgesteckten Rahmen von Institutionen und Regulierungen neu herausfordert.

Im Gegensatz dazu wird in einer weiteren Reihe von Beiträgen (alle in J./Oe.) das Eindringen von Öffentlichem in das Private thematisch. Barbara Thiessen etwa diskutiert Veränderungen des Privaten am Beispiel haushaltsnaher Dienstleistungen. Diese gehen mit neuen und komplexen sozialen Ungleichheiten einher und führen zur Notwendigkeit, neu zu bestimmen und zu regulieren, was als privat, öffentlich oder gesellschaftlich zu gelten hat. Ursula Müller weist in ihrem Beitrag zu häuslicher Gewalt und deren rechtlicher Regulierung auf eine damit verbundene Ambivalenz hin: auf die Gleichzeitigkeit von Hilfe und Kontrolle im Rahmen staatlicher Interventionen. Sabine Berghahn stellt insgesamt 
einen Komplexitätszuwachs bei der $\mathrm{Re}$ gulierung des Privaten fest und diskutiert dies am Beispiel verschiedener Rechtsgebiete wie der Reproduktionsmedizin, des Familienrechts oder der Arbeitsmarktpolitik. Unter anderem konstatiert sie dabei zunehmende staatliche Eingriffsinteressen in das Private aufgrund enger werdender finanzpolitischer Spielräume. In diesem Kontext wird mehrfach auch die normative Bedeutung des Privaten thematisch. Der Beitrag von Uta Meier-Gräwe beispielsweise fordert eine Aufwertung und stärkere Anerkennung der komplexen privat erbrachten Fürsorgeleistungen, die das künftige Humanvermögen der Gesellschaft absichern. Auch Christel Eckart befasst sich mit dem Thema Fürsorge und hebt die besonderen Beziehungsqualitäten hervor, die im Rahmen von Privatheit nicht nur möglich sondern zur Entfaltung von Identität und Autonomie auch erforderlich sind.

Wesentlicher analytischer Bezugspunkt verschiedener Beiträge im Band von Villa/ Thiessen ist demgegenüber die Frage nach Persistenz und Wandel von Familien(leit) bilden. Anhand einer Befragung von LehrerInnen zum Thema Kinderarmut zeigt Sabine Toppe die Wirkmächtigkeit des Leitbildes der Normalfamilie auf - private Kindheit und fürsorgeverantwortliche Mutter - und damit einhergehend die Privatisierung des Armutsthematik, was speziell zur Abwertung von Alleinerziehenden führt. Petra Bauer und Christine Wiezoreck fragen, welche Familienbilder in die professionelle Arbeit von SozialpädagogInnen einfließen und rekonstruieren auch hier das Leitbild der bürgerlichen (erziehungskompetenten) Familie als positive Hintergrundfolie sozialpädagogischer Interventionen. In einem Beitrag zu gleichgeschlechtlichen Elternpaaren zeigt Doreen Kruppa, wie selbst diese aufgrund der Vorgaben einer heteronormativen Geschlechterkultur und damit verbundener Familienbilder zu Anpassungs- und Ab- grenzungsleistungen genötigt werden und es auch hier keineswegs einfach ist, egalitäre Arbeitsteilungsarrangements zu etablieren.

Dass in gängigen Familienleitbildern vor allem auf Mütter fokussiert wird, zeigt sich auch in Beiträgen zum demografischen Diskurs. Sowohl Heike Kahlert als auch Thomas Etzemüller (beide in V./T.) arbeiten heraus, wie in der Problemwahrnehmung Frauen für die demografische Entwicklung verantwortlich gemacht werden: Letztlich geht es darum, dass die „richtigen“ Frauen wieder (mehr) Kinder bekommen, während Männer im Diskurs weitgehend keine Rolle spielen. Besonders plastisch kann dies Lena Correll (in V./T.) anhand von Interviews mit kinderlosen Frauen zeigen, die vor dem Hintergrund der Gleichsetzung von Frausein und Mutterschaft mit entsprechenden Anrufungen konfrontiert sind und in beständige Legitimationsnotwendigkeiten geraten. Ein ungewöhnliches Mittel zur Regulierung des „richtigen“ Zeitpunkts von Mutterschaft analysiert Anke Spiess (in V./T.) mit dem sogenannten Babysimulator. Diese lebensgroße computergesteuerte Babypuppe wird angewendet, um vor allem weiblichen Teenagern aus niedrigen sozialen Schichten die Verantwortung einer frühen Schwangerschaft bewusst zu machen. Spiess betont die disziplinierenden und verunsichernden $W$ irkungen, die mit einer Teilnahme an einem BabysimulatorExperiment einhergehen und bei den Betroffenen zu Erfahrungen wie Versagen und Isolation führen, womit letztlich ihre Marginalisierung verstärkt wird. Darüber hinaus befassen sich verschiedene weitere Beiträge (in V./T.) mit medialen Bildern und künstlerischen Brechungen von Mutterschaft. Auch sie zeigen, wie wirkmächtig tradierte Deutungsmuster von Mütterlichkeit noch immer sind. Insofern nehmen die Beiträge in diesem Kontext vornehmlich eine kritische Position ein 
und fokussieren auf Persistenzen trotz Wandlungsprozessen.

Auch der Blick auf Vaterschaft und Väterlichkeit schließlich fördert Ambivalentes zutage. Karin Flaake (in V./T.) untersucht die Beteiligung von Vätern bei geteilter Elternschaft und zeigt, dass dadurch paarinterne Aushandlungs- und Umdeutungsprozesse notwendig werden, die nicht nur Befreiung sondern auch Verunsicherung bedeuten. Notwendig wird insbesondere die Auseinandersetzung mit eigenen Geschlechterbildern. Gerade das Zusammenbringen von Väterlichkeit, Hausarbeit und Männlichkeit ist dabei subjektiv mit der Gefahr von Verweiblichung verbunden. Brüche und Widersprüche thematisiert auch Karin Schwiter (in V./T.). Anhand der Rekonstruktion von Vaterbildern junger Erwachsener arbeitet sie heraus, dass Anforderungen an Väter häufig in der Vaterfigur des sorgenden (Wochenend-)Vaters zusammen laufen, welche sich sowohl von der Figur des abwesenden Vaters als auch von der des Hausmanns absetzt. Anwesenheit im Sinne von emotionaler Nähe und Fürsorglichkeit geht im Bild des sorgenden Vaters einher mit gleichzeitig weiterbestehender Abwesenheit im Alltag. Fürsorge als wichtiger Bestandteil des Bildes von guter Vaterschaft scheint sich zunehmend durchzusetzen, wie auch der Beitrag von Heather Hofmeister u.a. (in. V.T.) zeigt. Allerdings sagt dies noch nichts über die gelebte Realität väterlicher Fürsorge aus. Eine besondere Gruppe von Vätern untersucht Barbara Rinken (in V./T.) mit den alleinerziehenden Vätern. Anhand von Interviewmaterial rekonstruiert sie deren Vorstellungen und Praxen und kommt zu dem Ergebnis, dass ihre Lebensrealität als Männer, die einen - z.T. unfreiwilligen - „Territoriumswechsel“ (ebd., 226) vollzogen haben, Veränderungspotentiale zur Auflösung dichotomer geschlechtsspezifischer Zuweisungen von Fürsorgearbeit und Erwerbstätigkeit birgt.
In ihrer Fülle an methodischen Zugängen und empirischen Erkenntnissen verdeutlichen beide Sammelbände, dass mit den Grenzverschiebungen zwischen Öffentlichem und Privatem sowie den Veränderungen von Mutterschaft und Vaterschaft wichtige und zugleich hochaktuelle Forschungsfelder in den Blick genommen werden. Beide Bände thematisieren damit wesentliche Aspekte derzeitiger gesellschaftlicher Wandlungsprozesse, die zentral auch mit dem Verhältnis der Geschlechter verbunden sind. Je auf ihre Weise machen sie aufmerksam dafür, dass das Private immer auch gesellschaftlich ist. Zugleich zeigen sie, wie fruchtbar ein interdisziplinärer Blick auf diese Fragen ist. Über die Geschlechterforschung hinaus wäre beiden Bänden eine breite Rezeption zu wünschen.

Karsten Kassner

Michael Bayer, Gabriele Mordt, Sylvia Terpe, Martin Winter (Hrsg.): Transnationale Ungleichheitsforschung. Eine neue Herausforderung für die Soziologie. Frankfurt am Main: Campus Verlag 2008,356 S., $€ 39,90$

Die soziale Ungleichheitsforschung gilt als eines der zentralen Felder der Soziologie. Dass der Kategorie Geschlecht neben Klasse und Ethnie eine zentrale Rolle für das Phänomen Ungleichheit zukommt, gilt mittlerweile - zumindest als empirischer Befund - als unumstritten. Daneben hat sich die Fachdebatte schwer damit getan, die spätestens seit den 1980er Jahren beobachteten Prozesse der Globalisierung und Transnationalisierung in ein adäquates Instrumentarium zu übersetzen. Zwar hat sich die Auffassung durchgesetzt, dass methodologisch der Begriff der Gesellschaft nicht mehr mit der nationalstaatlich 
verfassten Gesellschaft gleichzusetzen und folglich ein „methodologischer Nationalismus“ (vgl. 8) zu kritisieren ist. Aber im Mainstream der Soziologie der sozialen Ungleichheit stellen transnationale Ungleichheitsverhältnisse bislang vornehmlich ein Experimentierfeld für neue, quantitative Datenerhebungsverfahren dar, die in komparativer und tendenziell ökonomistischer Manier auf den Vergleich von Ungleichheitsstrukturen verschiedener Länder abzielen. Es mangelt an einer ausgereiften soziologischen und damit gesellschaftstheoretischen Theoriebildung, die dazu imstande wäre, nationale und internationale, vertikale und horizontale Ungleichheiten unter einem gemeinsamen analytischen Dach zu diskutieren.

Dies jedenfalls ist zunächst die Diagnose zur Lage des eigenen Faches, die der vorliegende, von Michael Bayer, Gabriele Mordt, Sylvia Terpe, Martin Winter herausgegebene Band zum Ausgangspunkt für die Diskussion nimmt. Er gliedert sich in drei Teile, in denen unterschiedliche Seiten des Themas - „Aspekte“, „Kategorien“" und "Sphären“" von transnationaler Ungleichheit - beleuchtet werden. Die meisten der Beiträge entstammen einer Tagung anlässlich der Emeritierung von Reinhard Kreckel an der Universität Halle-Wittenberg im Jahr 2006 und würdigen die Arbeiten eines Autors, der schon früh auf das theoretisch uneingelöste „Problem der globalen Ungleichheit" (23) hingewiesen hat. Markiert hat Kreckel damit nicht zuletzt eine Aufgabe, an der seit einigen Jahren auch die feministische Forschung arbeitet, so dass der Band trotz der wenigen versammelten Autorinnen auch instruktive feministische Perspektiven verspricht.

Ein Beitrag von Reinhard Kreckel eröffnet die Debatte. In zehn Thesen diskutiert er ,,die Bedingungen der Möglichkeit einer weltgesellschaftlichen Öffnung der Soziologie der sozialen Ungleichheit"
(23). Kreckel vermutet, dass eine „Rezeptionssperre" innerhalb des Faches eine Thematisierung sozialer Ungleichheit in weltgesellschaftlicher Perspektive verhindert (29). Dafür verantwortlich sieht er eine historische Wahlverwandtschaft zwischen der Entstehung der sozialwissenschaftlichen Ungleichheitsforschung und der Behandlung der sozialen Frage innerhalb nationalstaatlicher Grenzen. Zwar sei in den Köpfen die globale soziale Frage schon länger als Wunschbild präsent. Solange jedoch keine Bedingungen ausfindig zu machen seien, die dieser Idee einen wirklichen Boden gäben, könne die soziologische Debatte von sozialer Ungleichheit nicht ihre methodologische Engfuihrung auf den Nationalstaat überwinden. Entsprechend notwendig sei die Frage, ob sich gegenwärtig reale Tendenzen für eine Globalisierung der sozialen Frage und damit ein integrierter weltgesellschaftlicher Rahmen ausfindig lassen machen, auf den sich eine soziologische Weltungleichheitsforschung beziehen könnte. Daraufhin diskutiert Kreckel, ob sich eine neue, globale „Polanyi-Konstellation“ Bahn bricht (49): sich eine Welt-Mittelklasse herausbildet, die im Weltmaßstab eine mäßigende und ordnungsstabilisierende Rolle übernehmen könnte. Kreckel verneint dies zunächst, nicht zuletzt unter Rekurs auf die These Branko Milanovics, der das Verschwinden einer Welt-Mittelklasse diagnostiziert. Jedoch erkennt er die Möglichkeit zur Aufhebung der Rezeptionssperre durch die politische Entwicklung von neuen normativen weltsozialpolitischen Relevanz- und Handlungsrahmen, die durch internationale Organisationen wie die Vereinten Nationen und internationale NGOs befördert werden (vgl. 60 f.). Walter Müller und Steffen Schindler stellen „Überlegungen zur Milanovic-These über die internationale Einkommensungleichheit" an und unterziehen damit zugleich die Kreckelsche Polanyi-Frage einer Re- 
vision. Durch eine Analyse neuerer Daten zur Einkommensungleichheit und durch eine kritische Beleuchtung des methodischen Vorgehens von Milanovic kommen sie zu einer gegenteiligen These: Statt einer Entleerung der Mitte komme es im globalen Maßstab zu einem Wachstum hin zur Mitte. Volker Bornschiers anschließender Versuch, eine Kurzgeschichte der Ungleichheit im Weltsystem zu schreiben, die die Frage der materiellen Ressourcen im Zusammenhang mit der Verteilung demokratischer Mitbestimmung diskutiert, mag zwar ambitioniert sein, bleibt jedoch zu kursorisch und letztlich auf den Bereich der Einkommensungleichheit beschränkt. Stefan Hradil beschäftigt sich ebenfalls mit den Ursachen ungleicher Einkommensverteilung - vorrangig allerdings in Deutschland. Der Beitrag erweitert die für die Ungleichheitsforschung bedeutsame Theorie des Ökonomen Simon Kuznet um eine „Personenseite“ (153) und damit um eine soziologische Perspektive. Kuznets Überlegung, wonach Produktivitätsunterschiede ökonomischer Sektoren Einkommensungleichheiten begründen können, wird zwar zugestimmt, gleichwohl aber stellten Arbeitsplätze nur die eine Seite der Entstehung von Einkommensunterschieden dar. Die andere Seite werde von den Menschen, die für diese Arbeitsplätze zur Verfügung stünden, und ihrer Bildung und Qualifikation gebildet.

Die bis dahin deutliche Dethematisierung von Geschlechterverhältnissen wird im Teil II des Bandes durch den Aufsatz von Cornelia Klinger angenehm durchbrochen. Die Autorin schlägt eine integrierte Perspektive auf das Thema transnationale Ungleichheitsverhältnisse entlang der Differenzierungsachsen von Klasse, Ethnizität/Rasse und Geschlecht vor. Der methodologische Nationalismus habe nicht nur zur Ausblendung jener Dimensionen sozialer Ungleichheit geführt, die jenseits der Grenzen des modernen Nationalstaa- tes lägen, sondern auch jener, die innerhalb des nationalstaatlichen Territoriums, aber sozialtopologisch unterhalb der Aufmerksamkeitsschwelle angesiedelt seien (vgl. 160). Der Aufsatz fuihrt auf überzeugende Weise vor, dass die „Analyse von Klasse, Rasse, und Geschlecht (...) lahm ohne die globale Dimension - eine Analyse der globalen Dimension hingegen blind ohne die Kategorien Klasse, Rasse und Geschlecht" ist (161, Herv. i.O.). In einer bemerkenswert feinsinnigen und komplexen historischen Analyse der Zusammenhänge der „,topologischen Kategorien Haus-WeltStaat" in ihren Wechselwirkungen mit den „personalen Kategorien Klasse-Rasse-Geschlecht" (162) verdeutlicht Klinger, dass sich die bisher geltenden Konstellationen sozialer Ungleichheiten entgegen dem Anschein nicht etwa auflösen, sondern im Kontext globaler Migrationsbewegungen und ,work-“" und „,care chains“ einen Gestaltwandel durchlaufen (192).

Martina Löw korrigiert die Diskussion an anderer Stelle. Sie zeigt, dass eine einseitige theoretische Öffnung zur Weltgesellschaft Gefahr läuft, die Stadt als eigenständige Vergesellschaftungseinheit aus dem Erkenntnishorizont zu verlieren und somit das Verhältnis des Lokalen als eigenlogischem Ort mit spezifischen Erfahrungsqualitäten zum Globalen nicht präzise bestimmen zu können. Löws Beitrag kann auch als eine kritische Replik etwa auf die Beiträge von Bornschier und Hradil gelesen werden. Denn mit der Betonung der Qualität von Erfahrung und Deutung wird das grundsätzliche Problem quantitativer Forschungen verdeutlicht, dass die gewonnenen Ergebnisse nichts erklären, sondern bestenfalls Phänomene beschreiben können (vgl. 208).

Der Aufgabe, das Thema Generationenverhältnisse im Kontext transnationaler Ungleichheit zu diskutieren, widmet sich Reinhard Sackmann. Als folgenreich für die Weltgesellschaft habe sich die neoliberal 
inspirierte staatliche Forcierung privater kapitalstockfinanzierter Rentenversicherung erwiesen. Risikoreichere individuelle Lebensläufe seien nicht-intendierte Folgen von Veränderungen der Lebenslaufpolitik, insbesondere veränderter Alterssicherungssysteme.

Der dritte Teil des Bandes zum Thema "Sphäre(n)" sozialer Ungleichheit beschränkt sich auf die ökonomische Sphere des Marktes. Die Aufsätze von Johannes Berger und Thomas Hanf stehen in einem engen Diskussionszusammenhang. Berger geht davon aus, dass Ungleichheiten in privatwirtschaftlich organisierten Marktwirtschaften nicht durch den uneingeschränkten Wettbewerb und damit durch Prinzipien des Marktes selber entstehen, sondern durch Abweichungen vom „Idealzustand des Wettbewerbs“ (272). Ungleichheit der Einkommen sieht er begründet durch soziale Schließungen von Marktteilnehmern, die Renteneinkommen generieren. Demgegenüber belegt Hanf unter Rekurs auf Schumpeter, dass Innovationen als dem Markt immanente Mechanismen zu Einkommensungleichheit führen.

Die gouvernementalitätstheoretisch informierten Ausführungen von Ronan Shamir zum Verhältnis von Ökonomisierung und Moralisierung schließen den Band ab. Der inspirierende Beitrag legt dar, dass die Moralisierung des ökonomischen Handelns konstitutiver Bestandteil des neoliberalen Projekts geworden ist. Als solcher löst er nicht nur die epistemologische Unterscheidung zwischen Markt und Gesellschaft auf, der Begriff der moralischen Pflicht wird selbst in die Markt-Rationalität eingepasst.

Von einem feministischen Standpunkt aus betrachtet ist der Sammelband insgesamt enttäuschend. Zwar ist der grundsätzliche Versuch zu begrüßen, die Ungleichheitsforschung um die Perspektive globaler Ungleichheitsverhältnisse zu erweitern, erfreulich ist auch, dass es expli- zit Bezüge zwischen einzelnen Beiträgen gibt. Gemessen aber allein am eigenen Anspruch der HerausgeberInnen stellen sich Fragen. Welchen Anschluss das Thema transnationale Ungleichheitsverhältnisse etwa im dritten Teil findet, wird bestenfalls in dem durchaus spannenden Aufsatz von Shamir deutlich. Problematisch ist auch, dass im Gros der Beiträge die Diskussion auf das Thema Einkommensungleichheit beschränkt bleibt und der Blick eurozentristisch verengt ist. Mit spezifischem Fokus auf die Dimension der Geschlechterverhältnisse fällt auf, dass die Diskussion in weiten Teilen auf deren systematische Integration verzichtet. Abgesehen von dem wegweisenden Text von Cornelia Klinger wird, wenn überhaupt, Geschlecht als eines von vielen Merkmalen behandelt, mit dem Ausprägungen sozialer Ungleichheit auch im transnationalen Kontext in Verbindung gebracht werden können. Offensichtlich ist auch der tendenzielle Ausschluss feministischer und genderbezogener Arbeiten. Gerade aber der Aufsatz von Klinger macht klar, dass eine solide theoretische Rahmung transnationaler Ungleichheitsverhältnisse einer feministischen Grundlegung bedarf und die Kategorie Geschlecht kein beliebig additives Moment ist. Der Band selbst zeigt schließlich, dass es „Rezeptionssperren" nicht nur im Hinblick auf das Thema globale Ungleichheit gibt, die es zu überwinden gilt. Ein systematischer Einbezug feministischer Perspektiven, insbesondere der Intersektionalitätsforschung, feministischer postkolonialer Theorien wie auch der feministischen Ökonomie würde hier nicht nur eine Leerstelle füllen, sondern wäre für die Fortentwicklung einer Soziologie transnationaler Ungleichheit sicherlich produktiv.

Alexandra Rau 
Daniela Rastetter: Zum Lächeln verpflichtet. Emotionsarbeit im Dienstleistungsbereich. Frankfurt/New York: Campus Verlag, 2008, 321 S., € 34,90; Hildegard Maria Nickel, Hasko Hüning, Michael Frey: Subjektivierung, Verunsicherung, Eigensinn. Auf der Suche nach Gestaltungspotenzialen für eine neue Arbeits- und Geschlechterpolitik. Berlin: Edition sigma, 2008, 237 S., $€ 18,90$

Was haben Emotionsarbeit und die Suche nach Gestaltungspotenzialen fuir eine neue Arbeits- und Geschlechterpolitik miteinander zu tun? Emotionsarbeit ist eine spezifische Form von Arbeit, die innerhalb einer personenbezogenen Dienstleistung geleistet werden muss. Es wird verlangt, dass der/die Beschäftigte höchst subjektive Anteile wie beispielsweise Einfuhhlungsvermögen dazu einsetzt, KundInnen zufrieden zu stellen, sie zum Kaufabschluss zu bewegen und an das Unternehmen zu binden. Die eigenen Emotionen muss er/ sie dabei fest im Griff haben, d.h. Emotionen müssen reguliert werden. Eine zentrale Frage der ,Subjektivierungsdebatte' der Arbeits- und Industriesoziologie ist die Bedeutung der subjektiven Potenziale im Arbeitshandeln. Emotionsarbeit im Dienstleistungsbereich kann als eine besondere Form der betrieblichen Subjektivierung betrachtet werden. Nickel/ Hüning/Frey greifen die ,Subjektivierungsdebatte' ebenfalls auf und sehen in der Subjektivierung ein Potenzial, das für eine neue Arbeits- und Geschlechterpolitik genutzt werden könnte. Denn, so ihre These, die Subjektivierung der Beschäftigten lasse sich nicht auf betriebliche Vorgaben beschränken, sonder weise ,eigensinnig' über betriebliche Bedingungen und Anforderungen hinaus. Ein gemeinsamer Bezugspunkt der beiden Publikationen ist also die Subjektivierungsdebatte. In beiden Arbeiten wird von einer betrieblich indu- zierten und einer individuell induzierten Subjektivierung gesprochen.

Rastetter stellt fest, dass Studien zur ,Subjektivierungsdebatte' (z.B. Moldaschl,Voß) das Konzept der Emotionsarbeit nicht einbeziehen. Zudem würden in diesen Forschungen, wie auch in frühen Arbeiten zu Emotionsarbeit (z.B. Russel-Hochschild) die positiven Aspekte der Subjektivierung für die Person nicht thematisiert. Rastetter jedoch argumentiert: Die betriebliche Praktiken sind ,weniger Unterdrückung als vielmehr Chance zur eigenen Subjektformung" (12).

Nickel/Hüning/Frey orten durch ihre Untersuchung vor allem zwei inhaltliche Aspekte, die in der Diskussion über Subjektivierung nicht beachtet wurden: Zum einen der Aspekt der Unsicherheit (bzw. Vertrauen) und zum anderen der Aspekt des Eigensinns der Beschäftigten. In beiden Arbeiten wird betont, dass Subjektivität kein völlig neues Phänomen der Arbeit ist. Die subjektiven Anteile der Arbeitskraft haben sich von einem ,Störfaktor' hin zu einer Ressource im Arbeitsprozess entwickelt. Um die Arbeitsanforderungen zu erfüllen, kann und muss Subjektivität in die Arbeit eingebracht werden. Rastetter sieht Subjektivierung deshalb als Chance und als Zwang zugleich.

Beide Publikationen wählten männlich dominierte Bereiche für die (qualitativen) empirischen Studien: das Untersuchungsfeld bei Rastetter ist die Versicherungsbranche und bei Nickel/Hüning/Frey die Deutsche Bahn AG. Die Fragestellungen, der weitere theoretische Bezugsrahmen und die Ebenen der Analysen der beiden Publikationen gehen jedoch in unterschiedliche Richtungen. Rastetter fragt einerseits nach der Bedeutung von Emotionsarbeit für die Subjekte, nach ihren Strategien zur Emotionsregulation und ihrer Identitätsarbeit und andererseits danach, wie Emotionsarbeit als Arbeitsform gestaltet wird; d.h. nach den betrieblichen Strategien 
zur Formierung höchst subjektiver Potenziale. Der Begriff der Emotionsarbeit wurde durch die Studien von Arlie Russel Hochschild bekannt. Ihre Publikation „Das gekaufte Herz" verweist darauf, dass die Kontrolle über die Gefühle nicht mehr beim Individuum, sondern bei anderen liegt. Emotionsarbeit ist nach Hochschild zur Herstellung des ,richtigen Gefühls' nötig. Aber die Spannung zwischen dem, was gefühlt wird und dem, was gefühlt werden sollte, führt längerfristig zu emotionalen Dissonanzen. Sie argumentiert, wer seine Gefuihle ständig manipuliere, werde sich selbst fremd.

Mit dem Verweis auf neuere Forschungen (z.B. Zapf; Voswinkel) kritisiert Rastetter die Arbeit von Hochschild als kapitalismuskritisch und pessimistisch, u.a. weil sie die Gefahr der Entfremdung vom Selbst überbewerte. Emotionsarbeit wird überwiegend (u.a. von Hochschild) mit ,Frauenarbeit' verbunden. Durch die empirische Untersuchung im Außendienst eines Versicherungsunternehmens sucht Rastetter daher auch nach Antworten auf die Frage, wie Männer mit Emotionsnormen umgehen, die dem „Männlichkeitsbild der Gesellschaft widersprechen“" (45). Im Ergebnis stellt sie fest, dass es - wenn Männer weiblich konnotierte Aufgaben ausführen - zu einer „Uminterpretation“ (45) der Arbeit kommt.

Um zu zeigen, dass es für EmotionsarbeiterInnen psychisch notwendig ist, Identitätsarbeit zu leisten, zieht Rastetter die (sozial)psychologischen Ansätze der Identitätskonstruktion (Keupp) und der sozialen Identität (Tajfel/Turner) heran. Das Konzept der Identitätskonstruktion besagt, dass Identität keine persönliche Eigenschaft ist, sondern stets eine Feststellung, die jemand über sich selbst trifft. Identitätsarbeit besteht darin, die Erfahrungsfragmente in einen für sich selbst sinnhaften und stimmigen Zusammenhang zu bringen. Das Konzept der Sozialen Identität beschäftigt sich vor allem damit, wie Identitätsbedrohung reduziert und wie kollektive Identitäten internalisiert werden können.

EmotionsarbeiterInnen werden in Dienstleistungsunternehmen mittels Identitätspolitik konstruiert - so eine These von Rastetter. Identitätspolitik (Politik wird in Anlehnung an Foucault als Disziplinierung verstanden) leistet das Unternehmen, indem es z.B. Trainingsangebote bereithält. Diese enthalten vorgefertigte Identitätsbausätze, die selbstrelevante Versprechungen (Kohärenz, Anerkennung, Unsicherheitsreduktion, positiver Selbstwert) machen. Durch sie soll ein ,regelrechtes' Verhalten der Beschäftigten garantiert werden. Die Ergebnisse der Untersuchung von Rastetter zeigen, dass auf der individuellen Seite immense Anstrengungen der Beschäftigten nötig sind, ihr Selbstbild und ihre Selbstachtung durch Strategien der emotionalen Selbststeuerung und der Regulierung des inneren Befindens zu wahren. Aber ohne Maßnahmen der Unternehmen - so Rastetter - wäre Emotionsarbeit nicht möglich.

Nickel, Hüning und Frey gehen in der Publikation „Subjektivierung, Verunsicherung, Eigensinn“ der Frage nach, „ob und wie sich auf betrieblicher Ebene, Vermarktlichung' und 'Subjektivierung' der Arbeit zeigen und welche Folgen dies für die betriebliche Arbeitspolitik und die betrieblichen Geschlechterverhältnisse hat" (11). Sie fokussieren auf die Frage, wie eine neue Arbeitspolitik gestaltet werden könnte, die das Selbständigkeitsbedürfnis der Beschäftigten berücksichtigt. Eine verstärkt geforderte Subjektivierung könnte vom Unternehmen nicht intendierte Folgen haben, indem Momente eigensinniger Widerständigkeit' der Beschäftigten über betriebliche Vorgänge hinausweisen und den Blick auf eine, gesunde Balance von Arbeit und Leben richten. Mit dem Begriff ,Eigensinn' (leider führen die AutorInnen nicht an, ob sie den Begriff in 
Anlehnung an A. Lüdtke oder Negt/Kluge verwenden) wollen sie die Verbindung von Subjektivierung und Neuausrichtung der Arbeitspolitik, die das Leben außerhalb der Arbeit mit einbezieht, herstellen.

In der Auswertung der vier Teilunternehmen der Deutschen Bahn AG (Konzernleitung, KundenService Zentrum, DB JobService $\mathrm{GmbH}$, DB Zeitarbeit $\mathrm{GmbH}$ ) zeigte sich, dass „Unsicherheit eine zentrale Begleiterscheinung von Vermarktlichung und Subjektivierung“ (14) ist, denn ein Teil der Ungewissheit der unternehmerischen Gewinnerwartung wird an die Beschäftigten weitergegeben. Unsicherheit äußerte sich in Angst vor Arbeitsplatzverlust. Der ,Eigensinn' der Beschäftigten äußert sich, indem diese versuchten, ihre individuelle Existenz sowie den Lebensanspruch, Arbeit und Leben zu verbinden, zu sichern. Die subjektiven Bewältigungsformen von Unsicherheit hängen stark von konkreten betrieblichen Bedingungen und sozialstrukturellen Faktoren wie Alter, Qualifikation, Lebensform und Geschlecht ab. In Bezug auf die Geschlechterpolitik kommt die Studie zum Ergebnis, dass sich das Unternehmen verstärkt entlang einer Leistungs- und Verwertbarkeitslogik orientiere und von normativen, auf Gerechtigkeit bezogenen Handlungslogiken verabschiede. Eine Individualisierung von Geschlechtergleichstellung habe sich verfestigt. Die kollektiven Verständigungsprozesse über Gleichstellung sind durch ,Egalitätsmythen' (Funder) und relativ wenig Sensibilität für die Genderproblematik (auch bei den befragten InteressensvertreterInnen) erschwert.

Das betrieblich geforderte, eigenverantwortliche Handeln' könnte die Beschäftigten dazu befähigen, ,sich die relative Eigenständigkeit der gesellschaftlichen Rahmenbedingungen unternehmerischen Handelns theoretisch und praktisch anzueignen und sie in kollektiven Verständigungsprozessen zur Grundlage des auch widerständigen Selbstständigkeitsbedürfnisses in Arbeit und Leben zu machen“ (43). Ein organisierter kollektiver Reflexionsund Austauschprozess (auch zur Genderproblematik) zwischen den Beschäftigten sei sinnvoll, um eine Basis für die Neuausrichtung betrieblicher und gewerkschaftlicher Arbeitspolitik zu schaffen.

Beide Publikationen sind eine Bereicherung furr die theoretischen Diskussionen zum ,Wandel von Arbeit' und der Rolle des Subjekts in diesem Prozess insbesondere dadurch, als bislang wenig beachtete Aspekte der Subjektivierung zur Diskussion eingebracht werden. Will man einen Einblick in die Problematik der Identitätsarbeit von EmotionsarbeiterInnen und die betrieblichen Strategien zur Subjektivierung von Beschäftigten erhalten, ist die Publikation von Rastetter sehr empfehlenswert. Für eine Arbeit, die den Anspruch erhebt, sich explizit mit Emotionsarbeit in einem Männerberuf zu beschäftigen, wäre es wünschenswert gewesen, die Erkenntnisse der Geschlechterforschung systematisch einzubeziehen. Der empirische Befund, dass sowohl Führungskräfte wie Versicherungsagenten den Beruf als ,männlich' konstruieren, sie im Alltagsverständnis weiblich konnotierte Anforderungen als ,männliche uminterpretieren' (45) ist eine weitere Bestätigung für die (zwar komplexeren) theoretischen Erkenntnisse z.B. von Wetterer zu Berufskonstruktion und Geschlechterkonstruktion. Eine Auseinandersetzung mit den (neueren) theoretischen Überlegungen zu Maskulinität (Meuser; Hungerbühler) als kontextabhängige und relationale Kategorie hätte die Studie bereichern können.

Die Arbeit von Nickel/Hünig/Frey greift die Erkenntnisse und Theorien der feministischen Arbeitsforschung systematisch auf. Insbesondere auch AkteurInnen der Interessensvertretungen erhalten durch diese Publikation einige Anregungen (z.B. Organisieren eines kollektiven Reflexions- 
und Austauschprozesses) für ihre politische Arbeit. $\mathrm{Ob}$ und wie es in der Praxis jedoch gelingt, mit Eigensinn aber (noch) wenig Sensibilität für Gender-Fragen ausgestatteten InteressensvertreterInnen zu einer neuen (kollekiven) Arbeits- und Geschlechterpolitik zu gelangen, bleibt indes abzuwarten.

Edeltraud Ranftl

Cornelia Klinger, Gudrun-Axeli Knapp (Hrsg.): ÜberKreuzungen. Fremdheit, Ungleichheit, Differenz. Münster: Verlag Westfälisches Dampfboot 2008, 277 S., $€ 27,90$

Der von Cornelia Klinger und GudrunAxeli Knapp herausgegebene Band mit dem Titel Überkreuzungen, Ungleichheit, Differenz liefert einen entscheidenden Beitrag zur weiteren Entwicklung feministischer Theorie. Die unterschiedlichen Aufsätze des Sammelbands versuchen - oft programmatisch - zu klären, was unter dem so genannten Intersektionalitätsansatz $\mathrm{zu}$ verstehen ist und inwiefern die Analyse der auf unterschiedlichen Ebenen verorteten Geschlechterfrage eine Antwort auf die „Dethematisierung“ gesellschaftlicher Ungleichheitsstrukturen sein kann.

Das Buch ist in zwei Teile gegliedert. Im ersten Teil (Soziokulturelle Differenzen in der Konstitution von Subjektivität) sind die Artikel versammelt, die sich mit der Rolle beschäftigen, welche Geschlecht, Ethnie und Klasse bei Subjektivierungsprozessen spielen. Der zweite Teil (Gesellschaft - Ungleichheit - Differenz) besteht aus Artikeln, die sich eher mit der gesellschaftstheoretischen Relevanz von Intersektionalität befassen. Bei meiner Lektüre des Bandes möchte ich dem in dieser Gliederung implizierten Leitfaden nicht folgen. Stattdessen finde ich es interessanter, die his- torische und politische Kontextualisierung und das spezifische Theorieverständnis zu problematisieren, das den unterschiedlichen Analysen zugrunde liegt.

Der einführende Text von Kathy Davis erläutert den Entstehungskontext des Konzepts der Intersektionalität und dessen ortgebundene Entwicklung sehr deutlich. Eingeführt vom black feminism Anfang der 1980er Jahre in den Vereinigten Staaten sei das Konzept der Intersektionalität der Ausdruck einer politischen Absicht gewesen. Es sei dabei um die Thematisierung derVerkopplung von sexistischen (sex), sozialen (class) und rassistischen (race) Diskriminierungsstrukturen und um eine Rehabilitierung der Identitätspolitik gegangen. Rezipiert in der zweiten Hälfte der 90er Jahre vor allem in Großbritannien, aber auch in der BRD, in den Niederlanden und in Skandinavien im Zusammenhang mit der wachsenden Bedeutung von Migrationsphänomenen unterscheide sich der europäische Intersektionalitätsansatz von dem des black feminism in vielerlei Hinsicht. Abgesehen von den politisch und historisch motivierten Schwierigkeiten, die mit der Verwendung des Begriffs race im europäischen Kontext verbunden seien, sind nach der Analyse von Davis die Unterschiede zwischen dem amerikanischen und europäischen Konzept von Intersektionalität vor allem theoretischer Art. Sie beträfen die Natur der Kategorie der Intersektion, die Art der Überkreuzung und das Verständnis von politischer Subjektivität bzw. der Alternative zwischen identity und agency. Unterschiede, an denen Davis zufolge die Inkompatibilität der feministischen Theorie des black feminism und eines postmodernen Feminismus deutlich werden.

Interessanterweise spiegelt sich circa zehn Jahre später diese theoretische und politische Alternative in einer veränderten Form in der Debatte wieder, die um die Bestimmung des Intersektionalitätsansat- 
zes im deutschsprachigen Raum geführt wird und die in dem hier zu besprechenden Band - auch wenn er nicht nur Artikel von Autorinnen aus dem deutschsprachigen Raum enthält - präsentiert ist. Die Alternative, die hier dargelegt wird, ist die zwischen einem gesellschaftstheoretischen bzw. praxeologischen und einem dekonstruktivistischen bzw. systemtheoretischen Verständnis von Intersektionalität.

In ihrem Beitrag setzt Cornelia Klinger den Akzent auf die Natur der Kategorien, die in einem intersektionalen Ansatz in Betracht gezogen werden sollen. Es handele sich um Strukturkategorien, deren Zahl nicht willkürlich ausgedehnt werden könne und die nicht ausschließlich empirisch festgelegt werden könnten. Die Zentralität der Strukturkategorien Geschlecht, Ethnie und Klasse wird von Klinger in einer historisch-anthropologischen Perspektive begründet. Klasse und Geschlecht seien Strukturkategorien, weil sie sich auf die beiden Aufgaben bezögen, die grundsätzlich jede Gesellschaft zu lösen habe: „Zum einen die Herstellung und Verteilung der Mittel zum Leben, die Regelung der Produktion und Distribution von Gegenständen und Gütern aller Art; zum anderen [...] die Erzeugung und Erhaltung des Lebens selbst, die ,Reproduktion der Gattung', die Lebenshaltung und Lebensführung, die Regelung der alltäglichen menschlichen Beziehungen" (S. 42). Die Form der Produktions - und Distributionssphäre sowie die der Reproduktion der Gattung seien in der Moderne von einer spezifischen Art von Herrschaftsverhältnissen historisch bestimmt: der Herrschaft des Herrn über den Knecht, der Herrschaft des Mannes über die Frau. Die dritte Strukturkategorie ist die der Fremdheit, die Klinger im Allgemeinen aus der Gegenüberstellung von „sie/wir" hervorgehen lässt. In Zusammenhang mit der Formation der modernen Nationalstaaten gewinne diese dritte Strukturkategorie allerdings präzise historische Konturen: „Ethnizität ist die Antwort auf die erst mit dem Untergang des ancien régime virulent werdende Frage nach der Identität eines Volkes bzw. auf die Fragen, wie die Zugehörigkeit der Einzelnen zum nationalstaatlichen Ganzen bestimmt wird und wodurch sich die eine Nation von der/ den anderen unterscheidet. Ethnizität bildet die Grundlage des Nationalismus als der dritten spezifisch modernen Strukturkategorie neben Kapitalismus und Patriarchat" (S. 48-49).

Gudrun-Axeli Knapp zeigt, dass die Betrachtung von Klasse, Nationalität/Ethnizität, Geschlecht/Sexualität als Strukturkategorien der Europäischen Moderne einen gesellschaftstheoretischen Zugang im Sinne Adornos voraussetze. Im Unterschied $\mathrm{zu}$ Theorien, die geschlechtliche, nationale und soziale Verhältnisse als funktionale Differenzierungen der modernen Gesellschaft fassen, begreife die Gesellschaftstheorie sie als Ungleichheitsstrukturen. Trotz dieser Abgrenzungen von Theorien gesellschaftlicher Differenzierung (Spencer, Durkheim, Parsons bis hin zu Varianten des Neo-Funktionalismus und der Systemtheorie Luhmanns) plädiert Knapp für eine Öffnung der Ungleichheitstheorie, die in der Lage wäre, Differenzen innerhalb der Ungleichheitstrukturen zu berücksichtigen. Der Intersektionalitätsansatz erfülle diesen Anspruch, indem er zugleich die Ungleichheitsstrukturen und deren weitere Spezifizierung infolge bestimmter Überkreuzungen von Diskriminierungsprozessen ins Visier nehme.

Bei der Begründung des strukturellen Charakters der Kategorien ziehen Barbara Rendtorff und Regina Becker-Schmidt eine neue Dimension - die symbolische - in Betracht. Rendtorff tut dies, um darzulegen, inwiefern die Kategorie Geschlecht eine Differenz in Sinne Derridas darstelle, die nicht auf andere Ungleichheitstrukturen reduziert werden könne. Regina 
Becker-Schmidt geht es dagegen nicht um die Hervorhebung einer bestimmten Strukturkategorie, sondern um die Interferenz von symbolischer Ordnung und materieller Gewalt. In Anlehnung an die Freudsche Analyse der frühkindlichen Genese der Xenophobie, der zufolge die Mutter die erste Bezugsperson von Liebe und Hass sei, thematisiert Becker-Schmidt die Überkreuzung von Misogynie und Xenophobie auf der Ebene der Ich-Bildung.

Auch für Nina Degele und Gabriele Winker drücken die Kategorien der Intersektionalität strukturelle Herrschaftsverhältnisse aus. Die kapitalistische Gesellschaft voraussetzend sehen sie in der Reproduktion der Arbeitskraft (Strukturebene), in der symbolischen Reproduktion der sozioökonomischen Verhältnisse (Repräsentationsebene) und in derVerunsicherung der sozialen Akteurinnen (Identitätsebene) die grundlegenden Inhalte eines Intersektionalitätsansatzes, dessen Strukturprinzipien Klassismus, Heteronormativismus, Rassismus und Bodysmus seien. Das Heranziehen einer vierten Kategorie, der des Körpers, wird von Degele und Winker durch ihren praxeologischen Ansatz begründet, der von der widersprüchlichen Praxis sozialer Akteur_innen innerhalb gesellschaftlicher Felder ausgeht. Bei der Analyse von Konflikten oder Diskriminierungen, welche die Identitätsebene beträfen, erlaube die Betrachtung der körperlichen Dimension weitere spezifische Dominanzverhältnisse zu erfassen, die z.B. Alter, Attraktivität, Generativität und körperliche Verfasstheit umfassten.

Im Namen eines angeblich selbstverständlichen Konzeptes der „empirischen Offenheit" kritisiert Christine Weinbach die vermeintlich in den bisher dargestellten Beiträgen vorgenommene Fixierung von Kategorien als Strukturkategorien, die sie sowohl als ,a priori Festlegung“, als auch als historische Gesellschaftszuschreibung ablehnt, der zufolge in der Entwicklung der kapitalistischen und patriarchalischen Gesellschaft vom 19. Jahrhundert bis heute eine Kontinuität bestehe. Aus einer systemtheoretischen Perspektive betont Weinbach, dass Diskriminierungsphänomene heute nicht ohne Inklusionsmaßnahmen zu denken seien: „Familienverhältnisse weisen Anfang des 21. Jahrhunderts nur wenig Ähnlichkeit mit denen im 19. Jahrhundert auf und können nicht länger fraglos als ,bürgerlich-patriarchal' bezeichnet werden. Und der demokratische Nationalstaat basiert zwar nach wie vor auf einem ethnisch fundierten Volksverständnis; dieses stützt sich aber nicht länger auf eine exklusive Rassenlehre, sondern entwickelt sich in Deutschland seit einigen Jahren $\mathrm{zu}$ einem inklusiven, eher voluntaristisch orientierten Ethnizitätskonzept, das auf eine abstammungsorientierte Grundlegung verzichtet" (S. 173).

Birgit Sauer und Stefanie Wöhl ziehen in ihrem Beitrag Schlüsse aus den hier dargelegten unterschiedlichen Theorieverständnissen. Für sie geht der strukturtheoretische Strang der Intersektionalitätsdebatte davon aus, dass es distinkte Ungleichheitstrukturen gibt, die sich überschneiden. Für den antikategorialen bzw. dekonstruktivistischen Ansatz bestehe der Kern nicht in den Kategorien oder Achsen der Ungleichheit, sondern in der Interdependenz: Geschlechter-, Klassen- oder Ethnizitätsstrukturen entstehen und nehmen ihre Form und Gestalt erst in der Interdependenz an. In ihren Analysen über das Verhältnis von Antidiskrimierungs- und Diversitätspolitiken und Staat bemühen sich Sauer und Wöhl, wie schon Knapp, zu klären, inwiefern der strukturtheoretische und der dekonstruktivistische Ansatz nicht inkompatibel seien. Hier vermisse ich allerdings eine präzise Bestimmung dessen, wie beide Ansätze zu vereinbaren wären. Aus einer gesellschaftstheoretischen Perspektive gibt es kein Hindernis, weitere 
Differenzierungen von Ungleichheitstrukturen vorzunehmen. Umgekehrt aber ist m.E. nicht abzusehen, wie man aus einer „antikategorialen Perspektive“ zu einer historisch strukturellen Betrachtung von gesellschaftlichen Prozessen gelangen soll. Um diese Frage zu klären, wäre es nötig, die postmoderne Epistemologie zu überdenken.

Die Stärke des Buchs liegt nicht darin, eine Lösung für diesen theoretischen und politischen Konflikt gefunden zu haben, sondern darin, die verschiedenen Positionen in aller Deutlichkeit und begrifflichen Präzision darzulegen. In der Tat kann die weitere Entwicklung der feministischen Theorie eine Problematisierung dieser unterschiedlichen Theorieverständnisse nicht umgehen. Es geht in dem Buch letztendlich um eine kritische Auseinandersetzung mit dem Erbe der Gesellschaftstheorie und mit den Konsequenzen eines postmodernen Wissenschaftsverständnisses.

Rita Casale

Isabelle Stauffer: Weibliche Dandys, blickmächtige Femmes fragiles. Ironische Inszenierungen des Geschlechts im Fin de Siècle (= Literatur - Kultur - Geschlecht. Große Reihe, Bd. 50). Köln: Böhlau 2008, 351 S., $€ 44,90$

Im Zuge der gesellschaftspolitischen Umbrüche um die Jahrhundertwende bilden sich in der Literatur die Figuren von Femme fragile, Femme fatale, Dandy und Décadent heraus. Isabelle Stauffers Dissertation fragt auf Grundlage der zwischen 1913 und 1934 erschienenen Romane von Annette Kolb (1870-1967) und Franziska zu Reventlow (1871-1918) danach, wie sich Autorinnen mit diesen Figuren auseinander setzen.
Im Zentrum der Arbeit stehen die ,ironischen Inszenierungen des Geschlechts" um 1900. Ihre Konzentration auf die Rhetorik der literarischen Darstellung gründet Stauffer auf die Entscheidung, Figuren wie die Femme fragile als Topos zu lesen. Der Topos als katalogisierbares Argument unterstützt eine Beweisführung, die mittels ideologischer Gemeinplätze überzeugen will. In der Ironisierung werden diese Topoi gebrochen. Um diese Brechung zu untersuchen, fixiert Stauffer zuerst die grundlegenden Charakteristika, z.B. des Topos Femme fragile (80), um dann drei spezifische Techniken der Ironisierung zu ermitteln: Bruch, Mimesis und Metadiskurs.

Mit der Wahl dieser Geschlechtertopoi greift sie die dominanten und literaturwissenschaftlich bereits in grundlegenden Studien erforschten literarischen Figuren um 1900 auf. Neues bietet Stauffer dadurch, dass sie deren ironische Fraktur, auch in der genderüberkreuzenden Verwendung der Topoi beleuchtet.

Obwohl die Romane Kolbs wesentliche Elemente der Femme fragile verarbeiten, wie Stauffer beispielsweise anhand der Blumen-Metaphorik in Kolbs Das Exemplar zeigt, wird die Femme fragile durch die genannten rhetorischen Strategien ironisiert. Wo Kolb die Femme fatale einsetzt, wie in Daphne Herbst, wird die Dichotomisierung von fragiler und fataler Weiblichkeit selbst ironisch ausgestellt (vgl. 138). Kolbs exzessive Verwendung der Leseranrede in allen drei Romanen deutet Stauffer darüber hinaus als metadiskursive Ironie.

Reventlows monologischer Briefroman Von Paul zu Pedro hat nicht den topisch männlichen Dandy, sondern eine ,Femme Dandy“ zur Briefe schreibenden Hauptfigur. Wird der Dandy durch diese Verkehrung verfremdet, so heben die mimetische Übererfüllung des Dandytums sowie die Selbstironie der Briefeschreiberin die 
"Inszeniertheit der Geschlechtsidentität“ (170) hervor. Auch der Décadent wird in Reventlows Herrn Dames Aufzeichnungen und Der Selbstmordverein ironisiert. So wird etwa die topische Nervosität der Hauptfigur Herr Dame mimetisch übersteigert.

Stauffer stellt die Figurengestaltung der Romane detailliert dar, indem sie immer wieder auf die drei eingangs genannten Formen der Ironie rekurriert. Dies führt zu einem gewissen Schematismus, belegt jedoch ihre These, dass Kolb und Reventlow Geschlecht ironisch inszenieren. Stauffer bezieht sich hier auf Judith Butlers Theorie der Performativität von Geschlecht und das darin enthaltene Subversionspotential. Sie sieht Analogien zwischen den Textstrategien der Fin-de-Siècle-Autorinnen und Butlers Entwurf, favorisiert aber den Begriff der Ironie gegenüber dem der Parodie (220). Einzuwenden ist, dass gerade die Position ironischer Überlegenheit problematisch wird angesichts der immer schon vollzogenen Subjektivierung (subjectivation), die im Zentrum von Butlers Subjektformation steht.

Stauffer zeigt auf, wie die Figuren in den Romanen von Kolb und Reventlow Geschlechtergrenzen überschreiten und monolithische Identitätsformationen aufbrechen. Besonders hebt sie Reventlows Theatermetaphorik hervor und kommt so zu dem Ergebnis, dass die Autorinnen „lange vor Judith Butler [...] den Konstruktionsaspekt von Geschlecht sichtbar [machen]" (235). Stauffers Argumentation zielt mithin darauf ab, Kolb und Reventlow gendergeschichtlich aufzuwerten, indem sie zu Vorgängerinnen Butlers werden. Unberücksichtigt bleiben dagegen die Geschlechterdiskurse des frühen 20. Jahrhunderts. Gerade die rege feministische Publizistik um 1900 beschäftigt sich eingehend mit den Zuschreibungen von Weiblichkeit, zu denken ist hier z.B. an Rosa Mayreder oder Hedwig Dohm, und reagiert damit auf Theoretiker wie Otto Weininger.
Ebenso interessant wie das Ergebnis, dass Kolb und Reventlow Vorläuferinnen Butlers gewesen seien, wäre eine ergänzende Situierung der Autorinnen im vielfältigen Weiblichkeits- und Geschlechterdiskurs um 1900.

Die Konstruktion der Geschlechterbeziehungen in den Romanen analysiert Stauffer anschließend unter Rückgriff auf Barthes Fragmente einer Sprache der Liebe und Kristevas Geschichten von der Liebe. Mit Barthes zeigt sie die Ironisierung der Liebeskonstellationen bei Kolb auf und stellt die Autorin dadurch gleichzeitig in ein breites intertextuelles Bezugsfeld. Für Reventlow arbeitet sie im Unterschied dazu heraus, dass in ihren Romanen der feudale Liebescode den bürgerlichen ersetzt, welcher aus dieser Perspektive zum Objekt der Kritik wird.

Die vielfältige Ironisierung von $\mathrm{Ge}$ schlecht zeigt Stauffer anhand der ausgewählten Romane überzeugend auf. Wie die Autorinnen mit den gängigen $\mathrm{Ge}-$ schlechtertopoi umgehen, wird erhellend vermittelt, so dass Stauffers genaue Textlektüre als geradezu vorbildlich gelten kann. Gleichwohl ist der Blickwinkel der Arbeit begrenzt. Da der Fokus ausschließlich auf der Ironisierung von Geschlecht in den untersuchten Romanen liegt, bleiben die zahlreichen Schriften Kolbs, die sich auch mit der Geschlechterfrage befassen, weiterhin unbeachtet. Wie Stauffers rahmender Überblick zur Ironisierung von Geschlecht von Luise Gottsched bis Jeannette Winterson zeigt, ist ihr Anliegen die Platzierung der Autorinnen in einer Geschichte ironischen Schreibens. Angesichts der spärlichen Forschung zu Kolb und Reventlow hätte es gerade die historische Kontextualisierung ermöglicht, die Besonderheit beider Autorinnen deutlicher herauszuarbeiten. Das bleibt weiterhin ein Desiderat. 